Ann. Zootech., r965, 14 (2), r69-179.

\title{
MATURITE SEXUELLE ET APPARITION D'CEUFS A DOUBLE JAUNE ET SANS COQUILLE. RÔLE DU RÉGIME LUMINEUX ET DU NIVEAU ALIMENTAIRE
}

\author{
L. LACASSAGNE et J. P. JACQUET \\ Station de Recherches avicoles, \\ Centre national de Recherches zootechniques, Jouy-en-Josas (Seine-et-Oise)
}

\section{SOMMAIRE}

I84 poussins Rhode-Island $\times$ Wyandotle nés le ${ }_{5} 5$ décembre 1959 furent élevés sous 13 heures d'éclairement quotidien jusqu'à l'âge de 8 semaines. 4 lots furent alors constitués :

$\begin{array}{ll}2 & \text { lots témoins } \mathrm{T}_{3} \text { et } \mathrm{T}_{4} \\ 2 & \text { lots traités } \mathrm{L}_{3} \text { et } \mathrm{L}_{4}\end{array}$

Les témoins furent élevés sous lumière naturelle jusqu'au 27 septembre Ig60 puis recurent I4 $\mathrm{h}$ de lumière par jour. Les traités reçurent 6 heures de lumière artificielle par jour jusqu'à l'apparition du premier œuf puis une stimulation lumineuse croissante, une fois la ponte déclenchée.

Jusqu'à l'entrée en ponte le niveau alimentaire fut rigoureusement défini pour tous les lots et aligné sur la consommation du lot $\mathrm{L}_{\mathbf{4}}$ nourri ad libitum. Le lot $\mathrm{T}_{\mathbf{4}}$ reçut la quantité consommée par $\mathrm{L}_{4}$.

Les lots $\mathrm{L}_{3}$ et $\mathrm{T}_{3}$ reçurent $90 \mathrm{p}$. Ioo de la quantité consommé par $\mathrm{L}_{4}$.

Dans les conditions de l'expérience le régime lumineux de 6 heures retarde la maturité sexuelle de 17 à 2 I jours. Un abaissement de ro p. Ioo du niveau alimentaire n'a d'effet additif que dans les lots élevés en lumière naturelle. Dans ce cas la maturité sexuelle est retardée de 4 jours supplémentaires.

Dans chaque lot et durant les 6 premiers mois de ponte le pourcentage d'œufs d'un poids supérieur à $5^{\circ}$ grammes varie dans le même sens que le poids adulte des animaux de ces mêmes lots.

Le nombre d'œufs à double jaune, d'œufs à coquille faible et d'œufs sans membranes coquillières est en relation directe avec la maturité sexuelle. Le nombre de ces œufs anormaux est d'autant plus important que la maturité sexuelle est précoce.

Nous savons que des poulettes nées en décembre et élevées depuis leur naissance avec une durée quotidienne d'éclairement de 6 heures subissent un retard de maturité sexuelle si on les compare à des lots témoins élevés en lumière naturelle. Nous savons également que ces poulettes soumises à un régime lumineux restreint présentent durant leur croissance une consommation d'aliment nettement inférieure à celle des témoins - Bowman et Jones (I96r-I963) - Burmester et Card (I939) Cherry (I959) - Marr et coll. (I960) - LaCASSAgne et Jacouet (I963). 
A notre connaissance, la part respective du traitement lumineux et de la restriction alimentaire qui l'accompagne n'a jamais été déterminée dans le retard de la maturité sexuelle de telles poulettes. C'est le premier point que nous nous sommes proposé de préciser dans l'expérience qui suit.

Nous avons voulu en outre, vérifier nos résultats antérieurs sur les rapports entre la maturité sexuelle et certaines anomalies de l'ovogenèse : des poulettes à maturité sexuelle précoce pondent beaucoup plus d'œufs à double jaune que des poulettes identiques à maturité sexuelle retardée. Nous avons donc repris cette étude mais en l'étendant au contrôle des oufs pondus sans membrane coquillière ou simplement avec une membrane coquillière faiblement calcifiée (œufs hardés).

\section{CONDITIONS EXPÉRIMENTALES}

Cent-quatre-vingt quatre poulettes Rhode Island $\times W$ yandotte nées le I 5 décembre I959 furent élevées avec un éclairement quotidien de I3 heures de leur naissance à 1'âge de 8 semaines, puis, par distribution au hasard, réparties en 4 lots :

2 lots témoins $\mathrm{T}_{3}$ et $\mathrm{T}_{4}$

2 lots traités $\mathrm{I}_{3}$ et $\mathrm{I}_{4}$

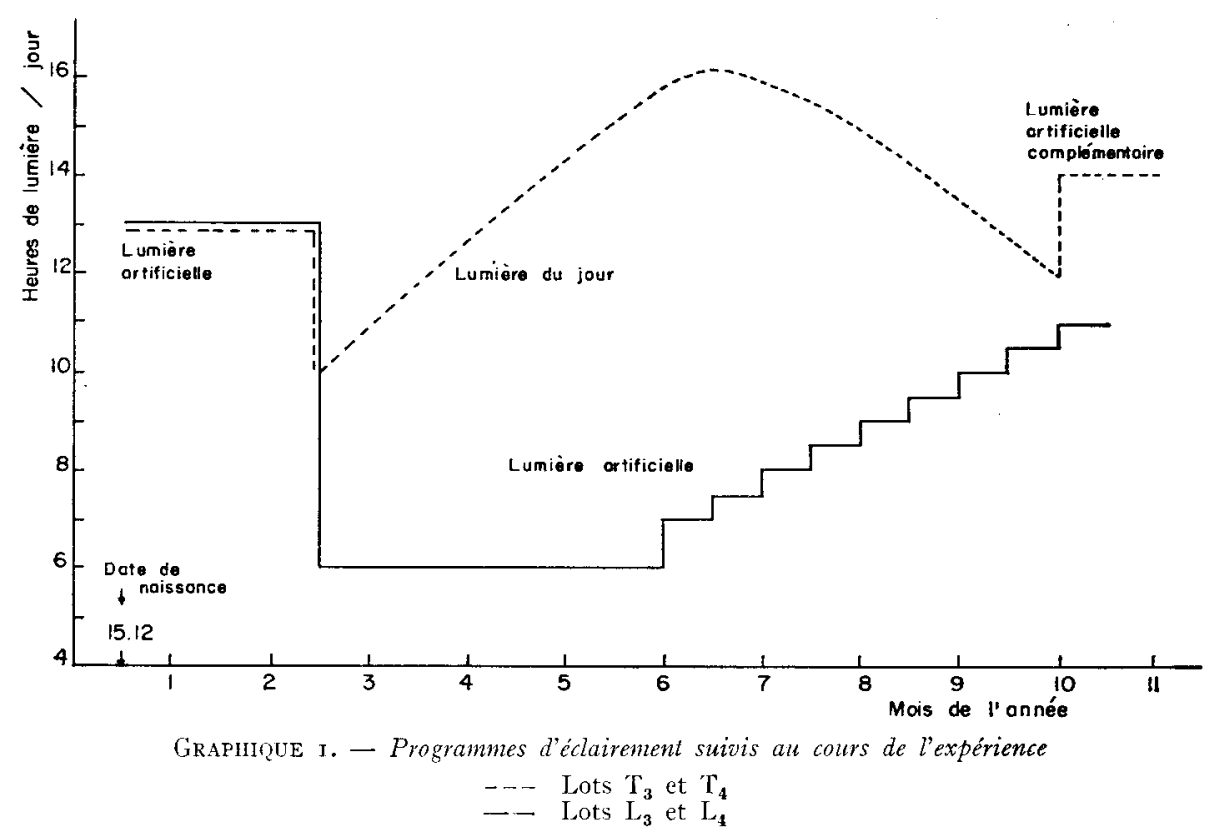

Les poulettes témoins furent élevées en lumière naturelle jusqu'au 27 septembre I960, puis reçurent un complément de lumière artificielle suffisant pour leur procurer au total I4 heures de lumière par jour.

Les lots traités furent éclairés artificiellement pendant 6 heures par jour de 
1'âge de 8 semaines à l'apparition du premier œuf. Une fois la ponte déclenchée la durée quotidienne d'éclairement fut portée à 7 heures durant ${ }_{5}$ jours puis augmentée de 30 minutes par quinzaine (graph. I).

Pendant la croissance, les lots de même traitement lumineux se trouvaient dans un même poulailler divisé en deux parties par un grillage. Les lots témoins étaient logés dans un poulailler isolé et pourvu de fenêtres. Les lots soumis au régime lumineux de six heures étaient logés dans un bâtiment identique mais dépourvu de fenêtres. Dans ce bâtiment la ventilation était mécanique et les entrées et sorties d'air munies de chicanes pour éviter la pénétration de la lumière.

Dès l'apparition du premier œuf dans un lot, les poulettes de ce lot étaient transférées en cages de ponte individuelles dans un bâtiment de mêmes caractéristiques.

A partir de l'âge de 8 semaines, les poulettes furent alimentées avec des quantités quotidiennes rigoureusement déterminées d'un aliment à 2050 calories productives par kilogramme et I 8 p. Ioo de matière azotée totale.

Dans les lots recevant 6 heures d'éclairement quotidien :

le lot $\mathrm{I}_{\mathbf{4}}$ fut alimenté ad libitum

le lot $\mathrm{L}_{\mathbf{3}}$ reçut go $\mathrm{p}$. Ioo de la quantité consommée par $\mathbf{L}_{\mathbf{4}}$

Dans les lots témoins :

le lot $\mathrm{T}_{\mathbf{4}}$ reçut une quantité égale à celle consommée par $\mathrm{L}_{4}$

le lot $\mathrm{T}_{3}$ reçut $90 \mathrm{p}$. Ioo de la quantité consommée par $\mathrm{L}_{4}$

A partir de l'entrée en ponte tous les lots furent nourris ad libitum.

Le poids des oufs fut relevé individuellement chaque jour et le poids moyen établi par quinzaine sur 4 jours consécutifs.

\section{RÉSULTATS}

\section{Consommation d'aliment}

La ration des lots $\mathrm{L}_{3}, \mathrm{~T}_{3}, \mathrm{~T}_{4}$ était établie chaque jour d'après la consommation du lot $I_{\mathbf{4}}$ le jour précédent, correction faite de la mortalité : le tableau I donne cette consommation pour le lot $\mathrm{L}_{4}$ par jour et par poulette de la $8^{\mathrm{e}}$ à la $23^{\mathrm{e}}$ semaine.

Les quantités de calories ingérées de la $8^{\mathrm{e}}$ à la $\mathrm{I} 6^{\mathrm{e}}$ semaine par le lot $\mathrm{L}_{4}$ correspondent aux quantités de calories ingérées, au cours d'une expérience similaire effectuée antérieurement, par des lots en éclairement naturel nés à la même date et nourris ad libitum avec un aliment à I 800 calories productives par kilogramme. Nous pouvons donc considérer que les lots $L_{3}$ et $T_{3}$ ont reçu une ration quantitativement inférieure d'environ ro p. roo à celle de lots élevés dans des conditions normales.

\section{Croissance des poulettes}

A partir de la $8^{\mathrm{e}}$ semaine une pesée individuelle de chaque animal fut effectuée tous les I 5 jours jusqu'à l'âge de II mois. Les résultats figurés au graphique 2 mettent en évidence trois périodes bien nettes dans la croissance pondérale: 
- une première période s'étend de la $8^{\mathrm{e}}$ semaines à la $19^{\mathrm{e}}$ semaine ;

- une deuxième période correspond à une croissance très rapide au moment de la maturité sexuelle.

- une troisième période débute lorsque l'intensité de ponte moyenne est de $50 \mathrm{p}$. Ioo et se manifeste par un ralentissement de la croissance.

Période I. - Quel que soit le régime lumineux fourni aux poussins et pour un même niveau alimentaire il n'existe pas de différence de croissance entre les lots $I_{4}, T_{4}$ et $I_{13}, T_{3}$ jusqu'à la $r g^{e}$ semaine :

- à consommation identique et traitements lumineux différents correspondent des croissances pondérales identiques;

- à un même traitement lumineux et à des niveaux alimentaires différents (restriction de Io $p$. IOO) correspondent des croissances très différentes $\left(\mathrm{L}_{3} \neq \mathrm{L}_{4}\right.$, $\left.\mathrm{T}_{3} \neq \mathrm{T}_{4}\right)$.

TABLEAU I

Consommation moyenne pour le lot 4

\begin{tabular}{c|c}
\hline \hline Age en semaines & $\begin{array}{c}\text { Quantité ingérée par poule } \\
\text { et par jour en grammes }\end{array}$ \\
\hline 8 à 10 & \\
10 à 12 & 52,7 \\
12 à 14 & 65,1 \\
14 à 16 & 80,6 \\
16 à 19 & 78,7 \\
19 à 21 & 83,7 \\
21 à 23 & 76,9 \\
& 85,3 \\
\hline
\end{tabular}

Période 2. - De la Ige semaine à 50 p. Ioo de ponte.

Le lot témoin $\mathrm{T}_{4}$ de la $\mathrm{Ig}^{\mathrm{e}}$ à la $2 \mathrm{I}^{\mathrm{e}}$ semaine et le lot témoin $\mathrm{T}_{3}$ de la $2 \mathrm{I}^{\mathrm{e}}$ semaine à $1 \mathrm{a} 23^{\mathrm{e}}$ semaine présentent une accélération de la croissance. Le même phénomène

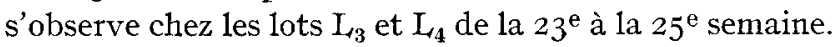

Cette augmentation de poids est en rélation pour chaque lot avec l'apparition de la maturité sexuelle.

Elle résulte vraisemblablement :

- en partie d'une augmentation de l'anabolisme correspondant au développement du système reproducteur de la poulette (MoRRIs et coll., I960) ;

- en partie d'une croissance compensatrice due au passage à tne alimentation ad libitum et au transfert des animaux en batterie.

Période 3. - Après $50 \mathrm{p}$. Ioo de ponte.

Lorsque l'intensité de ponte atteint 50 p. Ioo, tous les lots accusent un ralentissement de la croissance pondérale. Les lots $\mathrm{L}_{3}$ et $\mathrm{L}_{4}$, dont la maturité sexuelle est retardée présentent donc une période de croissance plus longue.

Il en résulte que pour une intensité de ponte de $50 \mathrm{p}$. roo leur poids corporel est supérieur à celui des lots non retardés $\mathrm{T}_{3}$ et ' $\mathrm{T}_{4}$.

Le poids adulte le plus élevé est celui du lot $\mathrm{T}_{4}$ non rationné et dont la période 
d'activité était limitée à 6 heures d'éclairement par jour. Par opposition, les poulettes ayant le poids adulte le plus faible se trouvent dans le lot $T_{3}$, restreint de Io p. Ioo et élevé en lumière naturelle.

\section{Maturité sexuelle}

Pour définir la maturité sexuelle de chaque lot nous avons choisi comme critères : l'âge de la poulette la plus précoce, l'âge moyen au premier œuf et l'âge du lot à une intensité de ponte moyenne de 50 p. roo déterminé comme le premier de deux jours consécutifs où la ponte atteint ou dépasse une intensité de 50 p. Ioo. Les données sont groupées au tableau 2 .

\section{TABLEAU 2}

Age des poulettes à la maturité sexuelle

\begin{tabular}{|c|c|c|c|}
\hline & $\begin{array}{c}\text { Age } \\
\text { de la poulette } \\
\text { la plus précoce } \\
\text { en jour. }\end{array}$ & $\begin{array}{l}\text { Age moyen } \\
\text { au premier œuf. }\end{array}$ & $\begin{array}{c}\text { Age } \\
\text { à } 50 \% \text { de ponte }\end{array}$ \\
\hline $\mathrm{T}_{3}$ restreint & 144 & $152,2 \pm 1,1^{*}$ & $15^{\prime}$ \\
\hline$T_{4}$ & 138 & $148,3 \pm 1,1$ & 150 \\
\hline $\mathrm{L}_{3}$ restreint & 159 & $168,3 \pm 0,8$ & 171 \\
\hline $\mathrm{L}_{4}$ & 159 & $169,0 \pm 1,3$ & 171 \\
\hline
\end{tabular}

* Par analyse de variance différences significatives à

$\mathrm{P}<0,0005$ entre $\mathrm{I}_{3}$ et $\mathrm{L}_{3}$

$\mathrm{P}<0,0005$ entre $\mathrm{T}_{4}$ et $\mathrm{L}_{1}$

$\mathrm{P}<0,05$ entre $\mathrm{T}_{3}$ et $\mathrm{T}_{4}^{2}$

non significatives entre L,3 et L4.

Les résultats traduisent une nette différence de précocité sexuelle suivant les régimes lumineux. Par rapport aux témoins en lumière du jour les poulettes en régime lumineux de 6 heures accusent un retard de $\mathrm{r} 6$ à $2 \mathrm{x}$ jours.

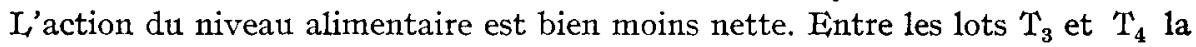
différence d'âge moyen au premier œuf n'est que de 4 jours (différence significative $\mathrm{P}<0,05$ ) et les lots $\mathrm{L}_{3}$ et $\mathrm{L}_{4}$ présentent une maturité sexuelle identique. Un abaissement de Io $\mathrm{p}$. Ioo de la quantité ingérée retarde donc légèrement la ponte des poulettes élevées en jours naturels mais n'a pas d'effets sur les lots élevés en régime lumineux de 6 heures.

\section{Intensité de ponte}

Le tableau 3 regroupe les intensités de ponte des 4 lots du premier œuf à la date du 22 octobre, soit durant 6 mois pour les lots $\mathrm{T}_{3}$ et $\mathrm{T}_{4}$ et durant cinq mois pour les lots $\mathrm{I}_{\mathbf{3}}$ et $\mathrm{I}_{\mathbf{4}}$. Les chiffres sont établis sur la totalité des œufs pondus par les 
poulettes, y compris les œufs sans coquille ou à coquille faible, sur la base du nombre de journées pondeuses.

La production d'œufs à 3II jours d'âge y est à l'avantage des poulettes rentrées en ponte hâtivement, les poulettes à maturité sexuelle retardée ayant un handicap

\section{TABLEAU 3}

Intensité de ponte par quinzaine au cours des 6 premiers mois de production

\begin{tabular}{|c|c|c|c|c|}
\hline Lots & $\begin{array}{c}\mathrm{I}_{3} \\
\text { Alimentation } \\
\text { restreinte }\end{array}$ & $\Gamma_{4}$ & $\begin{array}{c}\mathrm{L}_{3} \\
\text { Alimentation } \\
\text { restreinte }\end{array}$ & $\mathrm{L}_{4}$ \\
\hline Age moyen au $1^{\text {er }}$ œuf & 152 & $1 ' 8$ & 168 & 169 \\
\hline Période de contrôle & $\%$ & $\%$ & $\%$ & $\%$ \\
\hline 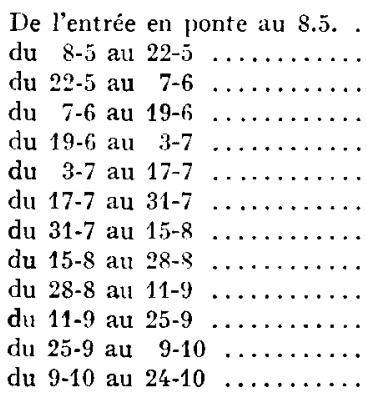 & $\begin{array}{l}3,0 \\
39,5 \\
75,1 \\
87,6 \\
91,5 \\
87,6 \\
80,6 \\
87,0 \\
84,1 \\
76,8 \\
73,3 \\
66,3 \\
69,5\end{array}$ & $\begin{array}{r}9,4 \\
53,4 \\
83,0 \\
91,2 \\
89,2 \\
88,8 \\
76,7 \\
76,8 \\
83,8 \\
76,6 \\
6,2,8 \\
60,9 \\
72,1\end{array}$ & $\begin{array}{l}6,0^{*} \\
29,8 \\
83,9 \\
85,3 \\
78,4 \\
75,9 \\
78,7 \\
80,8 \\
76,4 \\
80,2 \\
7 \times, 7 \\
77,8\end{array}$ & $\begin{array}{l}7,7^{*} \\
32,2 \\
68,8 \\
79,1 \\
80,7 \\
76,5 \\
75,8 \\
81,5 \\
82,6 \\
80,5 \\
78,4 \\
73,3\end{array}$ \\
\hline Intensité de ponte moyenne & 74,98 & 71,40 & 69,18 & 68,77 \\
\hline $\begin{array}{l}\text { Nombre d'œufs moyen } \\
\text { par poule à } 311 \text { jours d'âge. }\end{array}$ & $1 \geq 9,7$ & 129,2 & 114,1 & 113,4 \\
\hline Lots & \multicolumn{2}{|c|}{$\begin{array}{l}\text { Différence de précocité sexuclle } \\
\text { en jours }\end{array}$} & \multicolumn{2}{|c|}{$\begin{array}{l}\text { Différence de production } \\
\text { à } 311 \text { jours d'âge }\end{array}$} \\
\hline $\mathrm{T}_{3}-\mathrm{L}_{3}$ & \multicolumn{2}{|c|}{16 jours } & \multicolumn{2}{|c|}{15,6 œufs } \\
\hline $\mathrm{T}_{4}-\mathrm{L}_{4}$ & \multicolumn{2}{|c|}{21 jours } & \multicolumn{2}{|c|}{15,8 peufs } \\
\hline
\end{tabular}

* De l'entrée en ponte de $\mathrm{I}_{3}$ et $\mathrm{S}_{4}$ au $22-5$.

de 3 semaines. C'est pourquoi nous avons reporté dans le tableau 4 la production de chaque lot durant une période de I4I jours à partir de la date à laquelle fut atteinte pour chacun d'entre eux une intensité de ponte de $50 \mathrm{p}$. Ioo. Dans nos conditions expérimentales les intensités de ponte ainsi trouvées sont pratiquement identiques dans tous les lots surtout une fois déduits les cufs sans coquille ou à coquille faible. On peut se demander en effet si le léger avantage du lot ' $\mathrm{T}_{3}$ a une signification.

Remarquons le bon comportement des lots $T_{3}$ et $T_{4}$ malgré une date de naissance 
habituellement défavorable et malgré une chute très nette de la ponte du II septembre au 9 octobre due à l'absence d'un supplément de lumière artificielle à un moment où la décroissance de la longueur du jour est rapide.

\section{TABLEAU 4}

Production totale d'oufs en 1'1 jours de ponte à partir d'une intensité de ponte de 50 pour cent

\begin{tabular}{|c|c|c|c|c|c|c|c|}
\hline \multirow{2}{*}{ Lots } & \multirow{2}{*}{$\begin{array}{l}\text { Dates de } \\
\text { contrôle }\end{array}$} & \multicolumn{2}{|c|}{ Nombre de poules } & \multirow{2}{*}{$\begin{array}{l}\text { Nbr. d'œufs } \\
\text { par poule } \\
\text { (jour) }\end{array}$} & \multirow{2}{*}{$\begin{array}{c}\text { Nbr. d'œufs } \\
\text { par poule en } \\
\text { début } \\
\text { de période }\end{array}$} & \multirow{2}{*}{$\begin{array}{c}\text { Nbr. d'œufs } \\
\text { par poule jour } \\
\text { déduction faite } \\
\text { des oeufs sans } \\
\text { coquille }\end{array}$} & \multirow{2}{*}{$\begin{array}{l}\text { Nbr. d'œufs par } \\
\text { poule en début } \\
\text { de période } \\
\text { dèduction faite } \\
\text { des œufs sans } \\
\text { coquille }\end{array}$} \\
\hline & & $\begin{array}{c}\text { en début } \\
\text { de période }\end{array}$ & $\begin{array}{l}\text { en fin de } \\
\text { période }\end{array}$ & & & & \\
\hline $\mathrm{T}_{3}$ & $\begin{array}{l}d u 21-5 \text { au } \\
8-10 \text { inclus }\end{array}$ & 40 & 37 & 0,81 & 108,5 & 0,79 & 105,9 \\
\hline $\mathrm{T}_{4}$ & $\begin{array}{l}\text { du } 16-5 \text { au } \\
3-10 \text { inclus }\end{array}$ & 40 & 38 & 0,79 & 107,3 & 0,77 & 104,6 \\
\hline $\mathrm{I}_{3}$ & $\begin{array}{l}\text { du } 6-6 \text { au } \\
2 t-10 \text { inclus }\end{array}$ & 40 & 38 & 0,78 & 108,7 & 0,77 & 107,6 \\
\hline $\mathrm{I}_{4}$ & $\begin{array}{c}\mathrm{du} 6-6 \text { au } \\
24-10 \text { inclus }\end{array}$ & 40 & 36 & 0,78 & 102,4 & 0,77 & 100,9 \\
\hline
\end{tabular}

\section{Poids de l'ouf}

Le tableat1 5 donne le poids moyen par mois et le tableau 6 le pourcentage d'œufs d'un poids supérieur à 50 et 55 grammes pour les 22 premières semaines de ponte dans chaque lot.

\section{TABLEAU 5}

Poids moyen des oufs par mois de ponte (en grammes)

\begin{tabular}{|c|c|c|c|c|c|c|c|}
\hline & & \multicolumn{6}{|c|}{ Période de ponte } \\
\hline \multicolumn{2}{|l|}{ Lots } & $1^{\mathrm{er}}$ mois & $2 \mathrm{e}$ mois & $3^{e}$ mois & $4^{\mathrm{e}}$ mois & $5^{\mathrm{e}}$ mois & $6^{\mathrm{e}}$ mois \\
\hline \multirow{2}{*}{$\begin{array}{c}\text { En lumière } \\
\text { naturelle }\end{array}$} & $T_{3}$ & $46,5 \pm 0,6$ & $51,0 \pm 0,4$ & $54,2 \pm 0,3$ & $56,2 \div 0,4$ & $57,1 \pm 0,4$ & $60,1=0,3$ \\
\hline & $\mathrm{T}_{\mathrm{i}}$ & $46,8 \pm 0,8$ & $51,4 \pm 0,3$ & $53,4=0,3$ & $56,0 \pm 0,4$ & $58,4 \pm 0,2$ & $60,3=0,3$ \\
\hline \multirow{2}{*}{$\begin{array}{l}\text { A } 6 \text { heures } \\
\text { de lumière }\end{array}$} & $\mathrm{L}_{3}$ & $48,1 \doteq 0,5$ & $51,7 \pm 0,2$ & $54,4 \pm 0,2$ & $\tilde{\mathbf{5} 5}, 1 \pm 0,2$ & $57,0 \pm 0,2$ & $59,0 \pm 0,4$ \\
\hline & $\mathrm{L}_{4}$ & $49,3 \pm 0,5$ & $53,3 \stackrel{-1}{-} 0$, , $^{\prime}$ & $56,5+0,3$ & $58,4 \pm 0,2$ & $58,7 \pm 0,3$ & $61,6=0,4$ \\
\hline
\end{tabular}


Durant le premier mois de ponte les lots $I_{13}$ et $L_{4}$ présentent un poids d'œuf légèrement supérieur à celui des lots $\mathrm{T}_{3}$ et $\mathrm{T}_{4}$. Mais seul le lot $\mathrm{I}_{\mathbf{4}}$ maintient son avance durant les 22 semaines de contrôle. Ce poids d'œuf supérieur est en liaison.

\section{TABLEAU 6}

Réparition des oufs par classes de poids

(en pour cent).

\begin{tabular}{|c|c|c|c|c|c|c|c|c|}
\hline \multirow{2}{*}{\multicolumn{2}{|c|}{ Lots }} & \multicolumn{6}{|c|}{ Classes de poids en grammes } & \multirow{2}{*}{$\begin{array}{c}\text { Pourcentage } \\
\text { d'œufs de poids } \\
\text { ssupérieur à } 50 \mathrm{~g}\end{array}$} \\
\hline & & $<4$ & $45-50$ & $50-55$ & $55-60$ & $60-6 \overline{5}$ & $>65$ & \\
\hline \multirow{2}{*}{$\begin{array}{l}\text { En lumière } \\
\text { naturelle }\end{array}$} & $\mathrm{T}_{3}$ & 7,3 & 19,0 & $33, \dot{\prime}$ & 28,0 & 9,6 & 2,7 & 73,6 \\
\hline & $\mathrm{T}_{4}$ & 7,7 & 19,2 & $3: 3,5$ & 26,1 & 10,1 & $3,{ }^{\prime}$ & 75,3 \\
\hline \multirow{2}{*}{$\begin{array}{l}\text { A } 6 \text { heures } \\
\text { de lumière }\end{array}$} & $\mathrm{L}_{3}$ & 4,9 & 19,2 & 37,8 & 29,4 & 7,1 & 1,6 & 75,9 \\
\hline & $\mathrm{L}_{4}$ & 3,0 & 10,9 & 28,1 & 36,7 & 17,5 & 3,8 & 86,1 \\
\hline
\end{tabular}

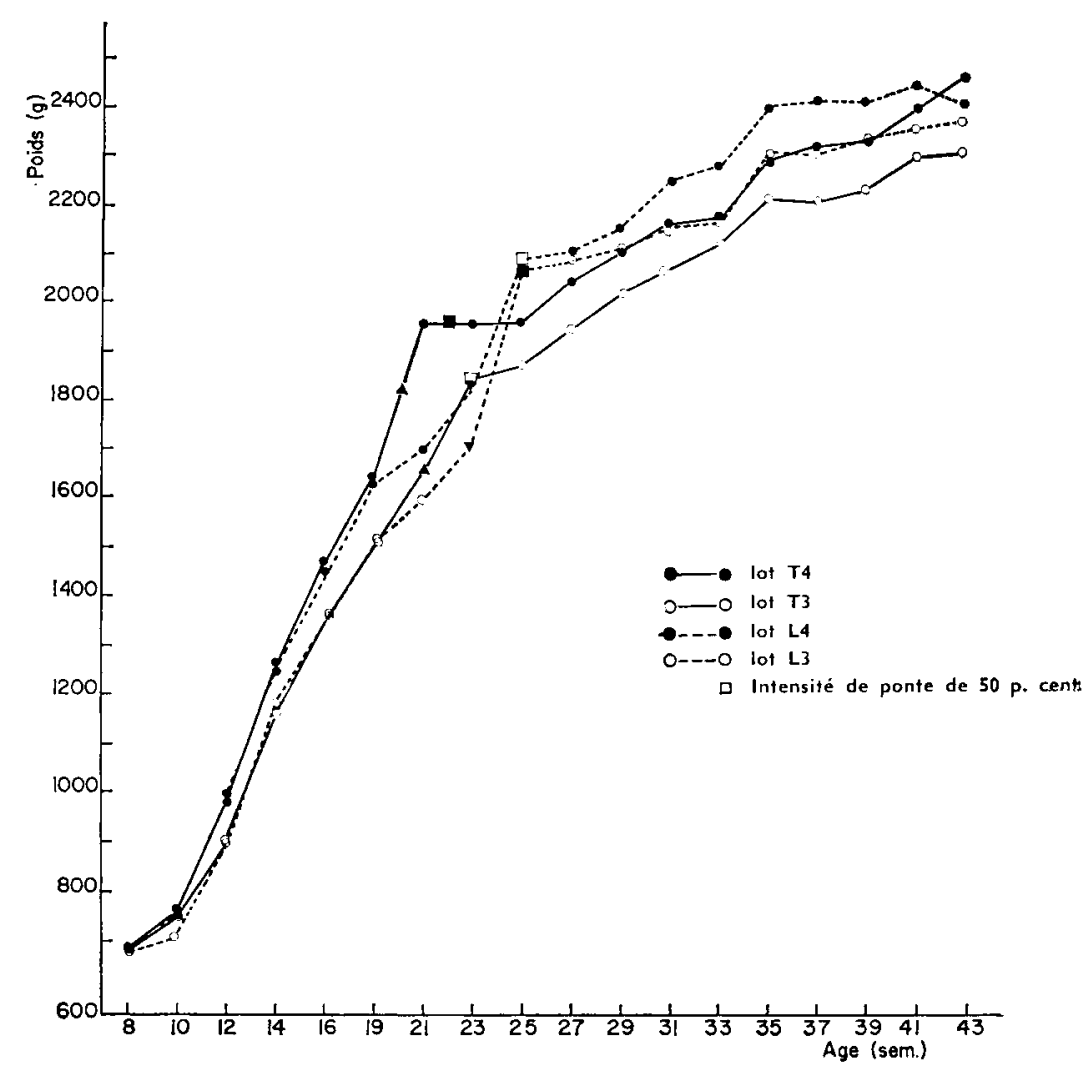

Graphique 2. - Poids moven des poulettes par lot de la $8^{\mathrm{e}}$ à la $43^{\mathrm{e}}$ semaine 
avec un poids corporel plus important durant toute la période observée. Une comparaison du tableau 6 et du graphique 2 nous montre d'ailleurs que le classement des lots par ordre de poids d'œuf décroissant correspond à leur classement (exprimé par le nombre d'œufs de poids supérieur à 50 grammes) par ordre de poids corporel adulte décroissant, à savoir $\mathrm{L}_{4}, \mathrm{~L}_{3}$ et $\mathrm{T}_{4}, \mathrm{~T}_{3}$. Mais il faut remarquer que les différences de poids d'œufs observées entre le lot $\mathrm{T}_{3}$ et les lots $\mathrm{L}_{3}$ et $\mathrm{T}_{4}$ sont faibles et ne sont mises en évidence que par le tableau 6.

\section{Anomalies dans la formation des cuifs}

Eufs à double jaune, œufs hardés, œufs sans membrane coquillière.

Pour chiffrer le pourcentage d'œufs à double jaune nous avons systématiquement cassé tous les œufs de chaque lot durant les trois premiers mois de ponte.

Comme nous le montre le tableau 7 le nombre d'œufs à double jaune est significativement plus grand chez les poulettes des lots $T_{3}$ et $T_{4}$ élevés en lumière du jour que chez les poulettes des lots $L_{3}$ et $L_{4}$. Leur nombre est étroitement lié à l'âge d'apparition du premier œuf, qu'il ait été modifié par le régime lumineux ou le niveau alimentaire. Nous retrouvons donc la relation précocité sexuelle, cufs à double jaune que nous avions déjà signalée dans une expérience précédente (LACASSAGNE et JACQUET, I963).

\section{TABLEAU 7}

Pourcentage d'oufs à double jaune et d'oufs hardès ou sans membrane coquillière

\begin{tabular}{|c|c|c|c|c|c|c|c|c|c|}
\hline \multirow{2}{*}{ Lots } & \multirow{2}{*}{$\begin{array}{l}\text { Age moyen aul } \\
\text { premier cuf }\end{array}$} & \multicolumn{2}{|c|}{$\begin{array}{c}\text { Premier mois de } \\
\text { ponte }\end{array}$} & \multicolumn{2}{|c|}{$\begin{array}{l}\text { Deuxieme mois } \\
\text { de ponte }\end{array}$} & \multicolumn{2}{|c|}{$\begin{array}{l}\text { Troisitme mois } \\
\text { de ponte }\end{array}$} & \multicolumn{2}{|c|}{$\begin{array}{l}\text { Signification des différences du } \\
\text { premier mois de ponte }\left(^{2}\right)\end{array}$} \\
\hline & & $\begin{array}{l}\mathrm{A}(\mathrm{l}) \\
(\mathrm{en} \%\end{array}$ & $\begin{array}{c}\mathrm{B}(\mathrm{I}) \\
(\mathrm{en} \%)\end{array}$ & $\left(\mathrm{en}^{\mathrm{A}} \%\right.$ & $($ en $\%)$ & $(\mathrm{en} \%)$ & en $\%)$ & A & B \\
\hline $\mathrm{T}_{3}$ & $152,2 \pm 1,1$ & 8,4 & 7,8 & 4,5 & 3,9 & 2,1 & 0,7 & $\begin{array}{l}\mathrm{T}_{3}-\mathrm{L}_{3} \\
\mathrm{P}<0,01\end{array}$ & $\begin{array}{l}\mathrm{T}_{3}-\mathrm{L}_{3} \\
\mathbf{P}<0,005\end{array}$ \\
\hline $\mathrm{T}_{4}$ & $148,3 \pm 1,1$ & 14,4 & 11,3 & 6,0 & 4,7 & 1,3 & 0,6 & $\begin{array}{l}\Gamma_{3}-\mathrm{T}_{4} \\
\mathrm{P}<0,001\end{array}$ & $\underset{\text { non significatif }}{\mathrm{T}_{3}-\mathrm{T}_{4}}$ \\
\hline $\mathrm{I}_{\mathbf{3}}$ & $168,3 \pm 0,8$ & 4,7 & 2,1 & 2,3 & 1,2 & 0,5 & 1,7 & $\stackrel{\mathrm{T}_{4}}{\mathrm{P}<0,0005}$ & $\begin{array}{l}\mathrm{T}_{4}-\mathrm{L}_{4} \\
\mathrm{P}<0,0005\end{array}$ \\
\hline $\mathrm{L}_{4}$ & $169,0 \pm 1,3$ & 4,8 & 3,3 & 1,6 & 1,1 & 0,7 & 2,6 & $\begin{array}{c}\mathrm{L}_{3}-\mathrm{L}_{4} \\
\text { non significatif }\end{array}$ & $\underset{\text { non significatif }}{\mathrm{L}_{3}-\mathrm{L}_{4}}$ \\
\hline
\end{tabular}

(1) A Eufs à double jaune

$B$ Qufs hardés ou sans membrane coquillière

(2) Méthode $\chi^{2}$

De plus, il apparaît une relation nette entre les œufs à double jaune et les œufs à coquille incomplète qu'il s'agisse d'œufs pondus sans membrane coquillière ou avec simplement une membrane coquillière faiblement calcifiée (graph. 3 et 4 ). Une matu- 


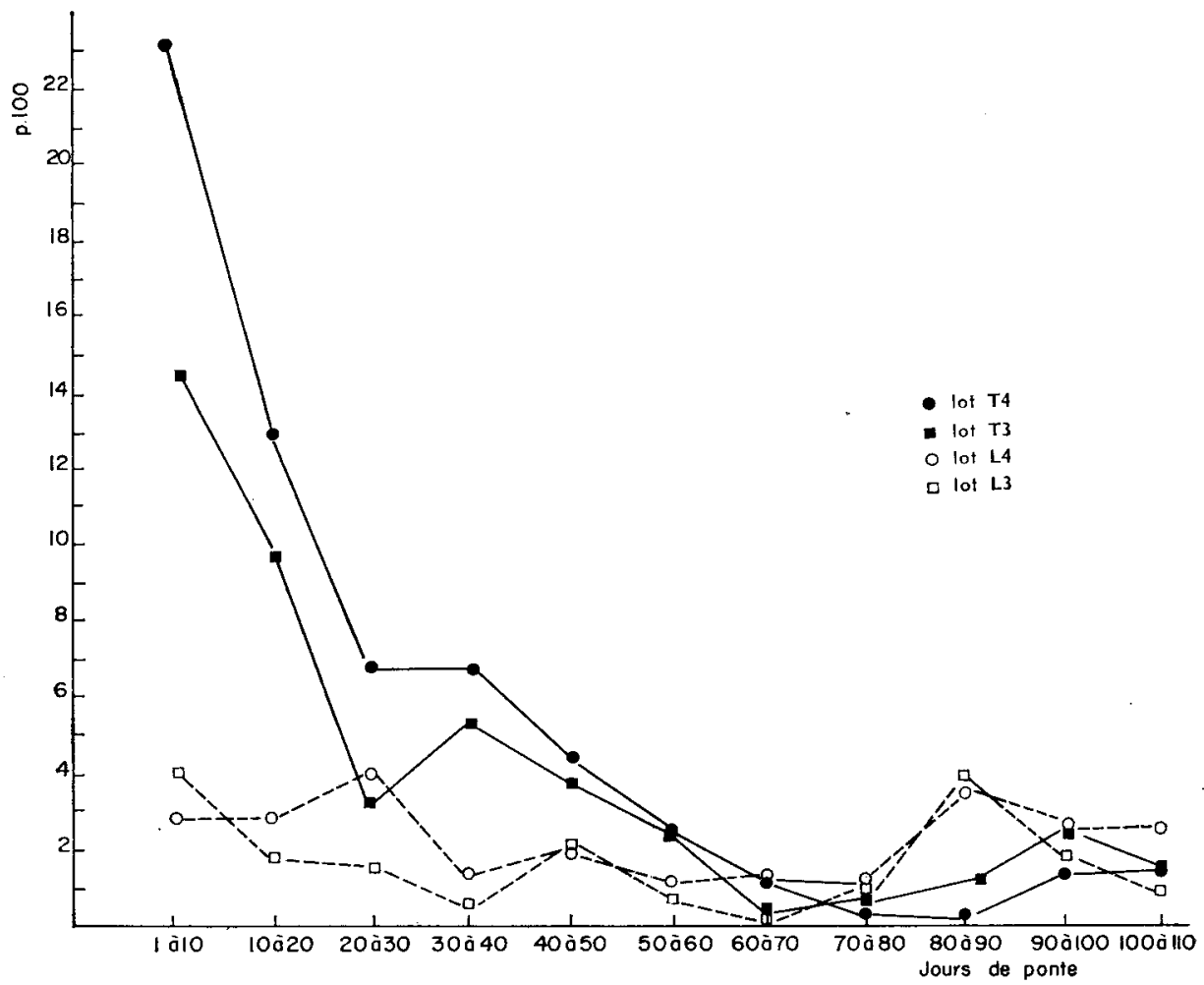

GRAPHIQUe 3. - Eriolution du nombre d'outs à double jaune durant les 3 premiers mois de ponte, exprimé en p. roo du nombre toial d'outs par période de to jours

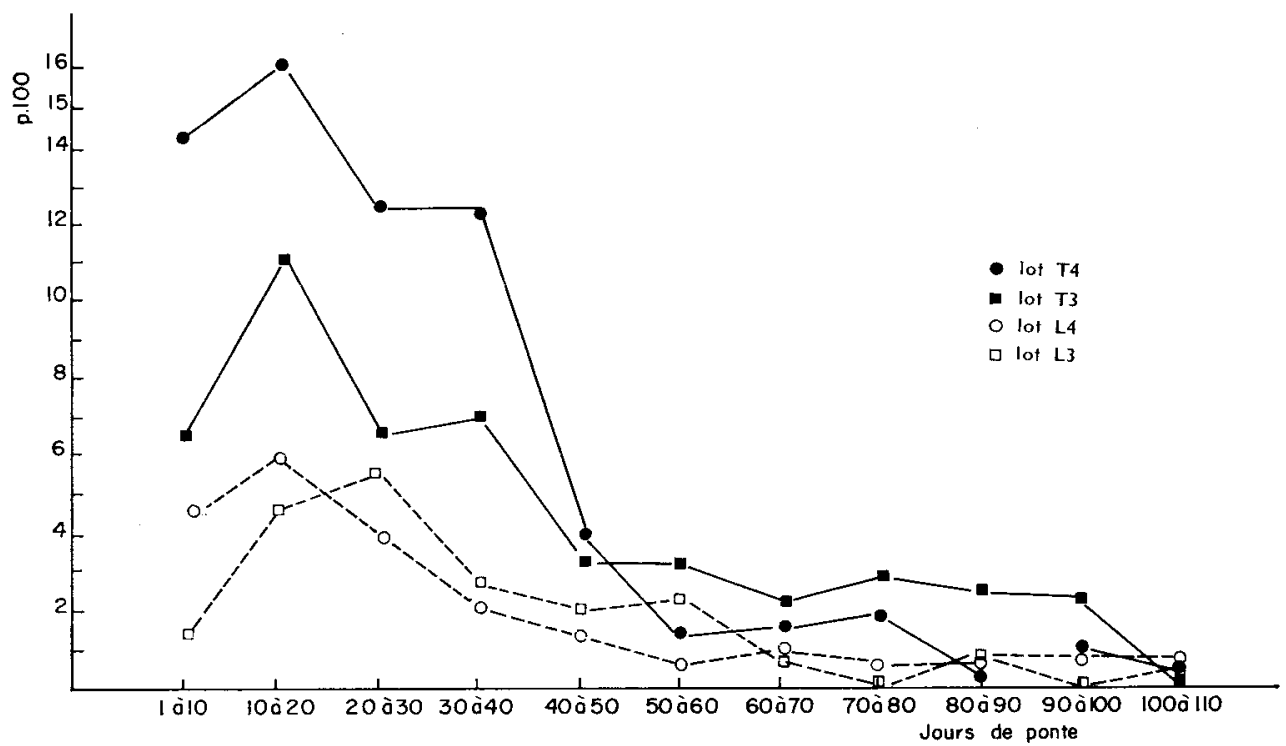

GraPHIQUE 4. - Évolution du nombre d'eufs hardés ou sans membrane coquillière durunt les 3 premiers mois de ponte, exprimé en p. 100 du nombre total d'auts par période de io jours 
rité sexuelle précoce s'accompagne donc de troubles de 1'ovulation aussi bien que de troubles de l'ovogenèse au niveau de la formation des membranes coquillières et de la calcification de la coquille.

Le nombre d'œufs perdus pour la commercialisation est ainsi respectivement de $I I 8$ et $I 3^{8}$ pour les lots $T_{3}$ et $T_{4}$, de 58 et 63 pour les lots $L_{3}$ et $I_{4}$ durant les r20 premiers jours de production. Soit de 2 à 3,5 oufs par poule en lumière naturelle contre I à I,5 pour les poules ayant une maturité sexuelle retardée.

$$
\text { Rę̧u pour publication en décembre } \mathbf{1 9 6 4 .}
$$

\title{
SUMMARY
}

\author{
SEXUAL MATURITY AND APPEARANCE OF DOUBLE-YOLKED \\ AND WITHOUT SHELL MEMBRANES EGGS. \\ ROLE OF LIGHT TREATMENT AND NUTRITIONAL LEVEL
}

I84 Rhode Island $\times$ Wyandotte chicks hatched on December $\mathrm{I} 5^{\text {th }}$, I959, were reared up to 8 weeks of age under 13 hours of daily lighting. 4 groups were then formed :

$$
\begin{aligned}
& 2 \text { control groups } T_{3} \text { and } T_{4} \text {, } \\
& 2 \text { treated groups } \mathrm{L}_{3} \text { and } \mathrm{L}_{4} \text {. }
\end{aligned}
$$

The control groups were reared under natural daylight until September $27^{\text {th }} 1960$; they were then given I 4 hours light per day. The treated groups were given 6 hours artificial light per day until the first egg had been laid in each group, and thereafter an increasing light stimulation once laying had started.

Until the start of the laying period, the nutritional level was strictly defined for all groups and adjusted according to the consumption of the $\mathrm{L}_{4}$ group fed ad libitum. The $\mathrm{T}_{4}$ group was pair fed with the $\mathrm{L}_{4}$ group.

The $\mathrm{L}_{3}$ and $\mathrm{T}_{3}$ groups were given 90 per cent of the amount of feed consumed by the $\mathrm{L}_{4}$ group.

Under the conditions of the experiment, the 6 hours light treatment delays sexual maturity by $I 7$ to $2 I$ days. A Io per cent decrease of the nutritional level has no additive effect except in the groups reared under natural daylight. In that case, sexual maturity was delayed by up to 4 more days.

In each group, and during the first 6 months of laying, the percentage of eggs weighing more than $5^{\circ} \mathrm{g}$ varies in the same direction as the adult weight of the birds in the same groups.

The amount of double-yolked eggs, thin-shelled eggs and eggs without shell membranes is directly related to sexual maturity. 'The earlier the sexual maturity, the higher is the amount of abnormal eggs.

\section{RÉFÉRENCES BIBLIOGRAFHIUES}

Bowman J. C., Jones R. H., ig6r. Lighting techniques for the domestic fowl. Brit. Poult. Sci., 2, 91-Io6. Bowman J. C., Jones R. H., 1963. Lighting techniques for the domestic fowl. Brit. Poult. Sci., 4, 27-37. Burmester B. R., Card L. E., I939. The effect of restricted feeding time on food intake, body weight and egg production. Poult. Sci., 18, 402-403. (Abstr.)

Cherry J. A., 1959. Restricted feeding time for the laying bird. World's Poult. Sci., 15, 371-377.

LACASSAGNe L., JACQUeT J. P., I963. Élevage de poulettes en lumière constante de six heures. Son action sur la croissance, la maturité sexuelle, le poids de l'œuf et le pourcentage d'œufs à double jaune en début de ponte. Ann. Zootech., 12, I59-172.

Marr J. E., Garland F. W., Pope C. W., Wilcke H. L., Bethike R. M., I96o. Further studies on controlled light during the growing and laying periods of chickens. Poult. Sci., 39, I272.

Morris T. R., Fox S., I96o. The use of lights to delay sexual maturity in pullets. Brit. Poult. Sci., I, $25-36$. 